\title{
Carnosic Acid Induces Antiproliferation and Anti-Metastatic Property of Esophageal Cancer Cells via MAPK Signaling Pathways
}

\author{
Sicong Jiang, ${ }^{1}$ Yinda Qiu, ${ }^{2}$ Zhaozhen Wang, ${ }^{3}$ Yulong Ji, ${ }^{4}$ Xiaofang Zhang, ${ }^{5}$ Xiaosong Yan, ${ }^{6}$ \\ and Zhiqiang Zhan ${ }^{7}$ \\ ${ }^{1}$ Division of Thoracic and Endocrine Surgery, University Hospitals and University of Geneva, Geneva 41211, Switzerland \\ ${ }^{2}$ School of Pharmaceutical Sciences, Wenzhou Medical University, Wenzhou, Zhejiang, China \\ ${ }^{3}$ Department of Clinical Medicine, Jiangxi Health Vocational College of China, Nanchang, Jiangxi, China \\ ${ }^{4}$ Jiangxi Key Laboratory of Translational Cancer Research, Jiangxi Cancer Hospital of Nanchang University, Nanchang, \\ Jiangxi, China \\ ${ }^{5}$ Department of Pathology, Jiangxi Cancer Hospital of Nanchang University, Nanchang, Jiangxi, China \\ ${ }^{6}$ Department of Thoracic Surgery, Jiangxi Cancer Hospital of Nanchang University, Nanchang, Jiangxi, China \\ ${ }^{7}$ Department of Oncology, Jiangxi Pingxiang People's Hospital, Pingxiang, Jiangxi, China
}

Correspondence should be addressed to Zhiqiang Zhan; zzq198265@163.com

Received 2 July 2021; Accepted 2 November 2021; Published 16 November 2021

Academic Editor: Yuan Seng Wu

Copyright (C) 2021 Sicong Jiang et al. This is an open access article distributed under the Creative Commons Attribution License, which permits unrestricted use, distribution, and reproduction in any medium, provided the original work is properly cited.

Background. Carnosic acid (CA) is a polyphenolic diterpene extracted from rosemary. Reports have shown that CA possesses anticancer activity. However, whether CA inhibits esophageal squamous cell carcinoma, an aggressive type of esophageal cancer, remains untested. Methods. The effects of CA on cell survival, migration, and apoptosis were evaluated by a combination of MTT, colony formation assay, flow cytometry, and Transwell assay. The potential signaling pathways involved were investigated via Western blot assay. Results. CA dose-dependently inhibited cell proliferation, apoptosis, migration, and colony formation. Mechanistically, CA arrested the cell cycle at G2/M phase, promoted cell apoptosis, induced DNA damage, and inhibited the MAPK signaling pathways. Conclusion. Our results suggest that CA is a potential anticancer drug for esophageal squamous cell carcinoma.

\section{Introduction}

Esophageal cancer is one of the most common malignant tumors in the digestive system. There are two main pathological types of esophageal cancer: esophageal adenocarcinoma (EAC) and esophageal squamous cell carcinoma (ESCC) [1-4]. The early vague and nonspecific symptoms of ESCC have contributed to its high morbidity and mortality [5]. Despite continuous improvement in treatment regimens, including surgery, chemotherapy, and radiotherapy, the 5-year survival rate remains fluctuating between $15 \%$ and $25 \%$ [6-8]. In addition, chemotherapeutic agents often have unbearable side effects. Thus, there is an urgent need to develop natural and safer compounds for the treatment of ESCC.
Carnosic acid (CA), a polyphenolic diterpene derived from the rosemary plant, possesses anti-inflammatory, antiviral, antioxidative, and antitumor activities [9]. Reports have shown that CA inhibited proliferation and migration of breast cancer and melanoma cells, arrested the cell cycle at the G2/M phase through suppression of cyclin A expression in leukemia and intestinal cancer, and induced apoptosis in prostate cancer and tumors of the human central nervous system $[10,11]$. This antitumor effect may be mediated through inhibition of the MAPK signaling pathways. This pathway represents a large family of serine/threonine kinases that, upon the reception of a stimuli, trigger a cascade of phosphorylation leading to specific cellular responses [12] and also plays a critical role in tumor progression and metastasis by induction of proteolytic enzymes that degrade 
the ECM (a key marker of invasion carcinoma), enhancement of cell migration, initiation of several prosurvival genes, and maintenance of tumor growth [13]. In addition, combination of CA with other drugs, such as curcumin, adriamycin, and carmustine, increased the antitumor effects of the latter compounds [14]. In this report, we studied the potential use of CA in the treatment of ESCC and explored the molecular mechanisms underlying its tumor-suppressive effect.

\section{Materials and Methods}

2.1. Cell Culture. Human esophageal squamous carcinoma cell line, KYSE-150, and normal human liver cells, MIHA, were obtained from the Institute of Biochemistry and Cell Biology, Chinese Academy of Sciences (Shanghai, China). KYSE-150 cells were cultured in RPMI-1640 (Gibco, Eggenstein, Germany) supplemented with $10 \%$ fetal bovine serum (FBS) (Gibco, Eggenstein, Germany) and $1 \times$ penicillin/streptomycin (Thermo Fisher Scientific, Carlsbad, CA, USA). MIHA cells were grown in Dulbecco's modified Eagle's medium (DMEM) (Gibco, Eggenstein, Germany) supplemented with $10 \%$ FBS and $1 \times$ penicillin/ streptomycin. All cells were cultured at $37^{\circ} \mathrm{C}$ in a $5 \% \mathrm{CO}_{2}$ atmosphere.

2.2. Cell Viability Assay. KYSE-150 (6500 cells/well) and MIHA (6000 cells/well) were seeded in 96-well plates. Cells were incubated overnight and treated with increasing concentrations of CA $(10,20$, and $40 \mu \mathrm{M})$ for $48 \mathrm{~h}$. The cell viability was determined using 3-(4,5-di-methylthiazol-2yl)-2,5-diphenyl-2 tetrazolium bromide (MTT) assay. In brief, cells were incubated with MTT reagent at $37^{\circ} \mathrm{C}$ for $4 \mathrm{~h}$, and $100 \mu \mathrm{l}$ DMSO was added to each well to dissolve formazan crystals. The absorbance was read at $490 \mathrm{~nm}$ on a spectrophotometer (DTX880, Beckman Coulter, CA, USA).

2.3. Colony Formation Assay. KYSE-150 cells were seeded at a density of 1000 cells/well in 12-well plates and allowed to attach overnight. Cells were treated with different concentrations of CA $(10,20$, and $40 \mu \mathrm{M})$ for 15 days at $37^{\circ} \mathrm{C}$. The cells were washed with PBS, fixed with $4 \%$ paraformaldehyde for $15 \mathrm{~min}$, and stained with crystal violet $10 \mathrm{~min}$ at room temperature. Colonies were counted using a stereomicroscope.

2.4. EdU Staining Assay. KYSE-150 cells were seeded in 6well plates the day before the experiment. Cells were treated with 10,20 , and $40 \mu \mathrm{M}$ CA for $48 \mathrm{~h}$. The EdU solution was added (final concentration of $10 \mu \mathrm{M}$ ), and cells were continued to incubate for $2 \mathrm{~h}$. Then, cells were processed for EdU staining using the EdU Proliferation kit (Beyotime, China) following the vendor's instructions. Cells were then observed under fluorescence microscopy (Nikon).

2.5. Cell Cycle Analysis. KYSE- 150 cells $\left(2.5 \times 10^{5}\right)$ were seeded in 6-well plates and incubated with 10,20, and $40 \mu \mathrm{M}$ CA for $48 \mathrm{~h}$. Cells were fixed in $70 \%$ ethanol for $24 \mathrm{~h}$, washed with cold PBS, and incubated with $50 \mu \mathrm{g} / \mathrm{mL}$ PI (propidium iodide) and $100 \mu \mathrm{g} / \mathrm{mL}$ RNase solution in PBS for $30 \mathrm{~min}$ at room temperature. Cells were analyzed on a flow cytometer (BD FACSalibur, BD Biosciences).

2.6. Cell Apoptosis Analysis. Apoptosis was analyzed using the apoptosis detection kit (BD Biosciences, USA) following the vendor's instructions. KYSE-150 cells were treated with CA $(10,20$, and $40 \mu \mathrm{M})$ for $48 \mathrm{~h}$ and washed with PBS once and Annexin V-binding buffer once. The cells were simultaneously incubated with fluorescein-labeled Annexin V and PI for $15 \mathrm{~min}$ at room temperature. Cells were then washed once again with Annexin V-binding buffer and resuspended with the same buffer, which were analyzed on a FACSCalibur (BD Biosciences, MD, USA).

2.7. Transwell Assay. Cells were treated with CA at 10, 20, and $40 \mu \mathrm{M}$ for $48 \mathrm{~h}$, which were then digested, resuspended, and diluted with serum-free medium to a concentration of $1 \times 10^{5} / 100 \mu \mathrm{l}$. Subsequently, $600 \mu \mathrm{l}$ of RPMI- 1640 containing $10 \%$ FBS was added into the lower chamber and cultured in a $37^{\circ} \mathrm{C}$ incubator. Cells on the top of the membrane were removed using Q-tips, and cells on the bottom of the membrane were fixed with $4 \%$ paraformaldehyde for $15 \mathrm{~min}$ at room temperature and washed with double distilled (DD) water. The cells were stained with crystal violet for $5 \mathrm{~min}$ and rinsed with DD water and 30\% glacial acetic acid to dissolve crystal violet. The plate was read at $560 \mathrm{~nm}$. Cell migration rate was calculated as follows: average OD of treated cells/average OD of control unit) $\times 100 \%$.

2.8. Immunofluorescence (IF) Assay. Cells on coverslips were fixed with $4 \%$ paraformaldehyde, permeabilized in $0.5 \%$ Triton X-100 (in $1 \times \mathrm{PBS}$ ), and incubated overnight at $4^{\circ} \mathrm{C}$ with primary antibody against 53BP1 (CST, Danvers, MA). Cells were washed three times and incubated with DyLight 549-conjugated anti-rabbit secondary antibodies for $1 \mathrm{~h}$ at room temperature. Cells were stained with DAPI, and images were acquired using Nikon Ti microscopy.

2.9. Western Blot Assay. Cells were grown on 6-well plates and were treated with 10,20 , and $40 \mu \mathrm{M} \mathrm{CA}$ for $48 \mathrm{~h}$. The cells were then washed with PBS and lysed using cell lysis buffer. The cell lysates were quantitated using the BCA method (BioRad, CA, USA). An equal amount of cell lysates was resolved on SDS-PAGE and electroblotted onto polyvinylidene difluoride membranes. The membranes were blocked using 5\% nonfat milk at room temperature for $2 \mathrm{~h}$ and incubated with primary antibodies at $4^{\circ} \mathrm{C}$ overnight. The membranes were incubated with the peroxidase-conjugated secondary antibodies for $1.5 \mathrm{~h}$ at room temperature. The immunoreactive bands were visualized using an ECL detection kit (BioRad Laboratories, CA, USA).

2.10. Statistical Analysis. All data were analyzed by GraphPad Prism 7.0. The means of multiple groups were compared by one-way analysis of variance, and the means of two groups 
were compared with the independent samples $t$-test. Values are expressed as the mean $\pm \mathrm{SD}$ of three independent experiments, with ${ }^{*} p<0.05,{ }^{* *} p<0.01$, and ${ }^{* * *} p<0.001$.

\section{Results}

3.1. Effects of CA Treatment on Cell Survival of KYSE-150 Cells. $\mathrm{CA}$ is a natural benzenediol abietane diterpene found in rosemary, with its chemical structure as shown in Figure 1(a). To evaluate the effect of CA on cell survival, KYSE-150 cells were treated with increasing concentrations of CA as indicated in Figure 1(b) for $48 \mathrm{~h}$ and cell viability was analyzed by MTT assay. CA at $<25 \mu \mathrm{M}$ had no significant effect on cell viability, which was decreased to $80 \%$ at $25 \mu \mathrm{M}$, and CA at $>25 \mu \mathrm{M}$ dose-dependently decreased cell viability. To evaluate the potency of CA, the half-maximal inhibitory concentration (IC50) was compared with that of the two most commonly used chemotherapeutic drugs, 5-fluorouracil (5-FU) and cisplatin (DDP). As shown in Table 1, the IC50 of CA for KYSE- 150 cells was $29.87 \pm 4.38 \mu \mathrm{M}$, compared to $65.98 \pm 1.39$ of $5-F U$ and $79.21 \pm 2.02$ of DDP and IC50 for MIHA cells was $>200 \mu \mathrm{M}$, compared to $>200$ of 5FU and $16.64 \pm 0.39$ of DDP. These results suggest that CA at less than $25 \mu \mathrm{M}$ had no obvious cytotoxicity and is a potent anticancer drug in KYSE-150 cells.

3.2. CA Inhibits the Proliferation of KYSE-150 Cells. To evaluate the effect of CA on colony formation capability, the colony formation assay was performed using KYSE- 150 cells as described in the Materials and Methods section. As shown in Figures 2(a) and 2(b), CA inhibited colony formation in a dose-dependent manner. To evaluate the effect of CA on cell proliferation, KYSE-150 cells were treated with EdU as described in the Materials and Methods section. As shown in Figures 2(c) and 2(d), CA dose-dependently inhibited cell proliferation.

3.3. CA Arrests Cell Cycle at the G2/M Phase. To further explore the molecular mechanisms of CA-induced inhibition of cell proliferation, KYSE-150 cells were treated with 10,20 , and $40 \mu \mathrm{M}$ of CA for $48 \mathrm{~h}$ and the cell cycle was analyzed by flow cytometry. As shown in Figures 3(a) and 3(b), CA arrested KYSE-150 cells at the G2/M phase in a dose-dependent manner. It is worth noting that CA treatment increased the percentage of cells at the G2/M phase and decreased the percentage of cells at the G0/G1 phase relative to controls. Cells were then treated with 5,10 , and $20 \mu \mathrm{M}$ of $\mathrm{CA}$, and $\mathrm{G} 2 / \mathrm{M}$ phase-related proteins were evaluated by Western blot. CA dose-dependently decreased the expression of cyclin B1, MDM2, and CDC2 (Figure 3(c)). Taken together, CA-induced inhibition of cell proliferation occurs most likely through suppression of G2/M phase-related proteins, leading to cell cycle arrest at the G2/M phase.

3.4. CA Provokes Strong DNA Damage Response. DNA damage induced by anticancer drugs can block cells at the G2/M phase, which prevents cells from entering into mitosis, leading to their anticancer effects. To investigate whether CA-induced G2/M phase arrest was caused by DNA damage, KYSE-150 cells were treated with CA for $48 \mathrm{~h}$ and P53 binding protein 1 (53BP1), a widely used marker for DNA double-strand breaks, was evaluated by immunofluorescence (IF) assay. As shown in Figure 4, CA dose-dependently increased the number of 53BP1-positive foci, with $\mathrm{CA}$ at $40 \mu \mathrm{M}$ reaching $\sim$ eight 53BP1-positive foci per nucleus. When phosphorylation of the Ser-139 residue of the histone variant $\mathrm{H} 2 \mathrm{AX}(\gamma-\mathrm{H} 2 \mathrm{AX})$ occurs, a molecular marker of DNA double-strand break was evaluated by Western blot. CA dose-dependently increased the expression of $\gamma$-H2AX. The results suggested that CA provokes a strong DNA damage repair response in KYSE-150 cells.

3.5. CA Induces Apoptosis of KYSE-150 Cells. Severe DNA damage usually leads to cell cycle arrest and apoptosis. To test this, KYSE-150 cells were treated with CA as indicated in Figure 5, and flow cytometry assay was performed to analyze the cell apoptosis. As expected, CA-induced KYSE-150 cell apoptosis in a dose-dependent manner (Figure 5(a)). When apoptosis-related proteins, $\mathrm{Bax}, \mathrm{Bcl} 2$, and cleaved caspase- 3 were analyzed by Western blot, CA dose-dependently decreased the expression of $\mathrm{Bcl} 2$ and simultaneously increased the expression of both Bax and cleaved caspase-3, respectively (Figure 5(c)). These results indicated that CA induces cell apoptosis by regulating the expression of apoptosisrelated proteins.

3.6. CA Inhibits Metastasis and Invasion of KYSE-150 Cells via Suppressed MAPK Signaling Pathway. Cell migration and invasion play an important role in cancer metastasis. To evaluate the effects of CA on cell migration and invasion, KYSE- 150 cells were treated with CA at 10,20 , and $40 \mu \mathrm{M}$ for $24 \mathrm{~h}$ and Transwell assay was performed. As shown in Figures 6(a) and 6(b), CA dose-dependently decreased cell migration. Recent studies have shown that inappropriate activation of the epithelial-to-mesenchymal transition (EMT) is associated with increased tumor invasion and metastasis $[15,16]$. To explore whether CA inhibits EMT, KYSE-150 cells were treated with CA and Western blot assay was used to determine the expression of the key proteins involved in the EMT process. However, as shown in Figure 6(c), CA did not inhibit tumor cell metastasis and invasion via an EMT pathway. To investigate the potential singling pathways involved in CAinduced inhibition of cell proliferation and migration, components of the signaling pathways were analyzed by Western blot. As shown in Figure 6(d), CA dose-dependently decreased the expression of p-ERK, p-JNK, and p-38 but did not affect that of total JNK, p-38, and ERK. These results suggest that CA inhibits cell metastasis and invasion through suppression of ERK, JNK, and p-38 signaling pathways.

\section{Discussion}

ESCC is one of the most aggressive cancers and ranked the sixth leading cause of cancer death in the world [17]. Although progress has been made in clarifying the molecular 


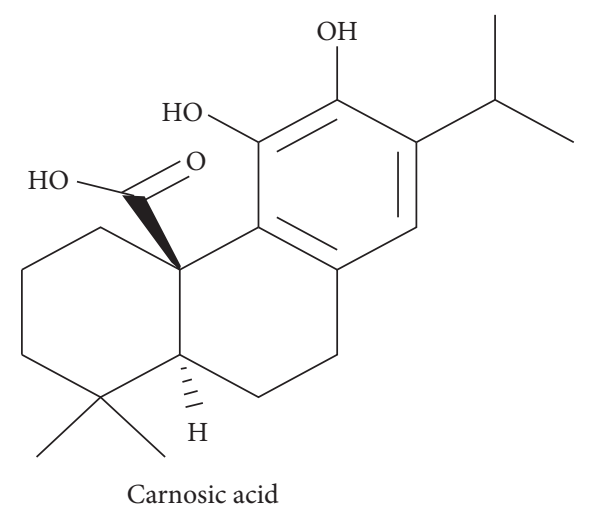

(a)

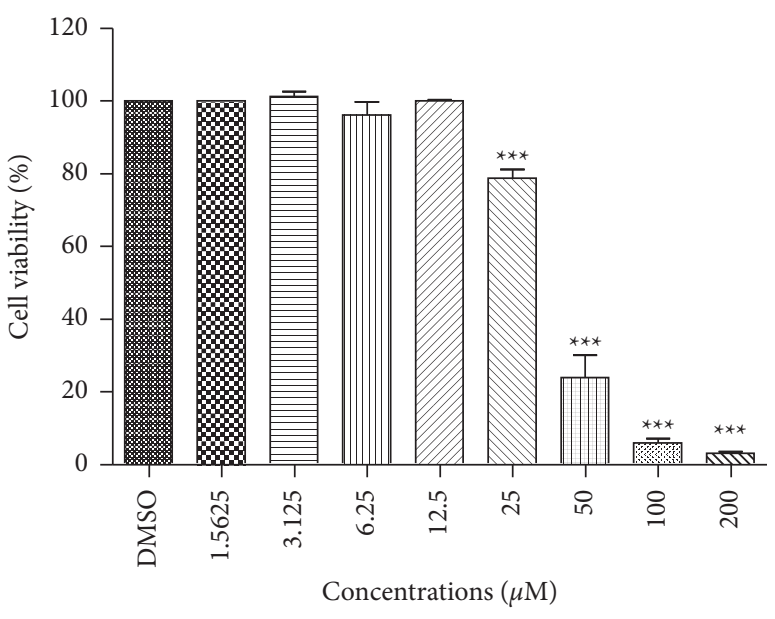

(b)

Figure 1: Evaluation of cytotoxicity and potency of CA. (a) The chemical structure of carnosic acid. (b) KYSE-150 viability was assessed using the MTT assay $48 \mathrm{~h}$ after treatment. Data were the means \pm SD of three independent experiments $\left({ }^{* * *} p<0.001 \mathrm{vs}\right.$ control group).

TABLE 1: IC50 $(\mu \mathrm{M})$ values were determined via the MTT assay.

\begin{tabular}{lcr}
\hline Cell lines/compounds & \multicolumn{2}{c}{ IC50 $(\mu \mathrm{M})$} \\
& MIHA & KYSE-150 \\
\hline Carnosic acid & $>200$ & $29.87 \pm 4.38$ \\
5-Fluorouracil & $>200$ & $65.98 \pm 1.39$ \\
DDP & $16.64 \pm 0.39$ & $79.21 \pm 2.02$ \\
\hline
\end{tabular}

IC50 $(\mu \mathrm{M})$ values were drug concentrations necessary for $50 \%$ inhibition of cell viability. Data are presented as "mean \pm SD" from at least three independent experiments in triplicates. The drug treatment period was $48 \mathrm{~h}$.

mechanisms of its pathogenesis, ESCC still carries high mortality rates, early metastasis, and poor prognosis $[18,19]$. CA is a functional ingredient of rosemary that has been used as an antioxidant food for many years [20, 21]. Recently, research has shown that CA exhibits antitumor activity in colon cancer, breast cancer, and skin tumors through inhibition of cell proliferation, invasion, and metastasis and induction of apoptosis and ROS production [22]. In the present study, we demonstrated that CA suppressed the malignant phenotypes of ESCC cells. Mechanistically, the tumor-suppressive effect of CA was mediated mainly by cell cycle arrest at the G2/M phase, promotion of severe DNA damage and apoptosis, and activation of MAPK multiple signaling pathways.

As a naturally occurring anticancer compound, CA has been studied for its cytotoxic effect in normal cells and its anticancer activity in different tumors. CA was demonstrated to decrease cell viability in a dose-dependent manner, with an IC50 of 25.6 to $96 \mu \mathrm{M}$ in breast cancer MCF7 cells and $19.6 \mu \mathrm{M}$ and $22.9 \mu \mathrm{M}$ in prostate cancer cell lines LNCaP and 22Rv1 cells, respectively [23, 24], suggesting it is a very potent anticancer compound. In agreement with the previous studies, we found that CA at less than $25 \mu \mathrm{M}$ did not have obvious cytotoxicity, and concentrations larger than $25 \mu \mathrm{M}$ dose-dependently decreased cell viability of an ESCC cell line, KYSE-150 (Figure 1(b)).

CA has been shown to inhibit the malignant phenotypes of cancer cells in different cancers, and its molecular mechanisms seem to be cancer type-dependent. CA has been reported to inhibit cell proliferation through cell cycle arrest at the G0/G1 phase in melanoma cancer [25], at the G2 phase in human glioma [26], and at the G1 phase in estrogen receptor (ER)-negative human breast cancer cells [27]. This anticancer activity was mediated by the capabilities of CA to activate p21-mediated signaling pathway [25] and induced apoptosis and production of reactive oxygen species (ROS) [28], inhibited the EMT [29], enhanced the anticancer effects of other compounds [26], and sensitized TRAIL-mediated apoptosis [30]. Here, we showed that CA dose-dependently inhibited the malignant phenotypes, including cell proliferation, migration, and colony formation, in an ESCC cell line, KYSE-150 (Figure 2). In addition, CA dose-dependently decreased the expression of G2/M phase-related proteins, MDM2, cyclin B1, and CDC2 (Figure 3). Anticancer drug-induced DNA damage can activate p53-dependent pathways, blocking the cell cycle at the G2/M phase and inhibiting cell proliferation [31-33]. Consistently, we demonstrated that CA could induce severe DNA damage, showing a dose-dependent increase in P53BP1-positive foci per nucleus after CA treatment (Figure 4), which was corroborated by an increased expression of $\gamma$-H2AX, a molecular marker of DNA double-strand break. It is wellknown that cells with irreversible DNA damage and cell cycle arrest will undergo apoptosis $[34,35]$. In line with this, we observed that CA dose-dependently increased KYSE-50 cell apoptosis compared with a nontreated control group by flow cytometry analysis (Figures 5(a) and 5(b)). These data showed a dose-dependent decrease in the expression of apoptosis-inhibiting factor, $\mathrm{Bcl} 2$, and a simultaneous increase in the expression of apoptosis-promoting factors, Bax and cleaved caspase-3, by Western blot (Figure 5(c)). MAPK signaling pathways play an important role in the initiation of tumor cell invasion and metastasis [36]. Indeed, CA dosedependently inhibited cell migration (Figures 6(a) and 6(b)), which was most likely mediated by the suppression of ERK, p-38, and JNK signaling pathways (Figure 6(d)). 


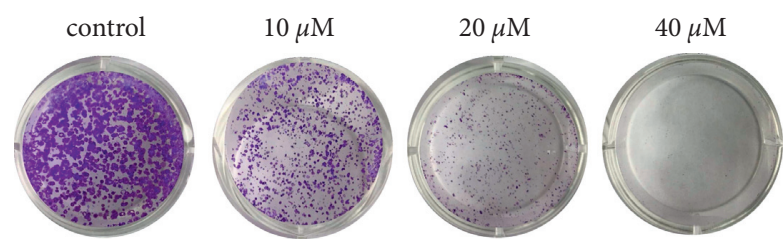

(a)

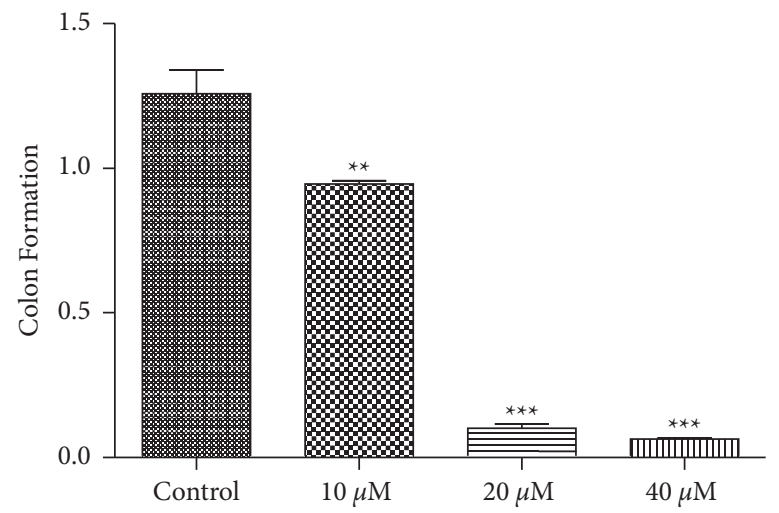

(b)

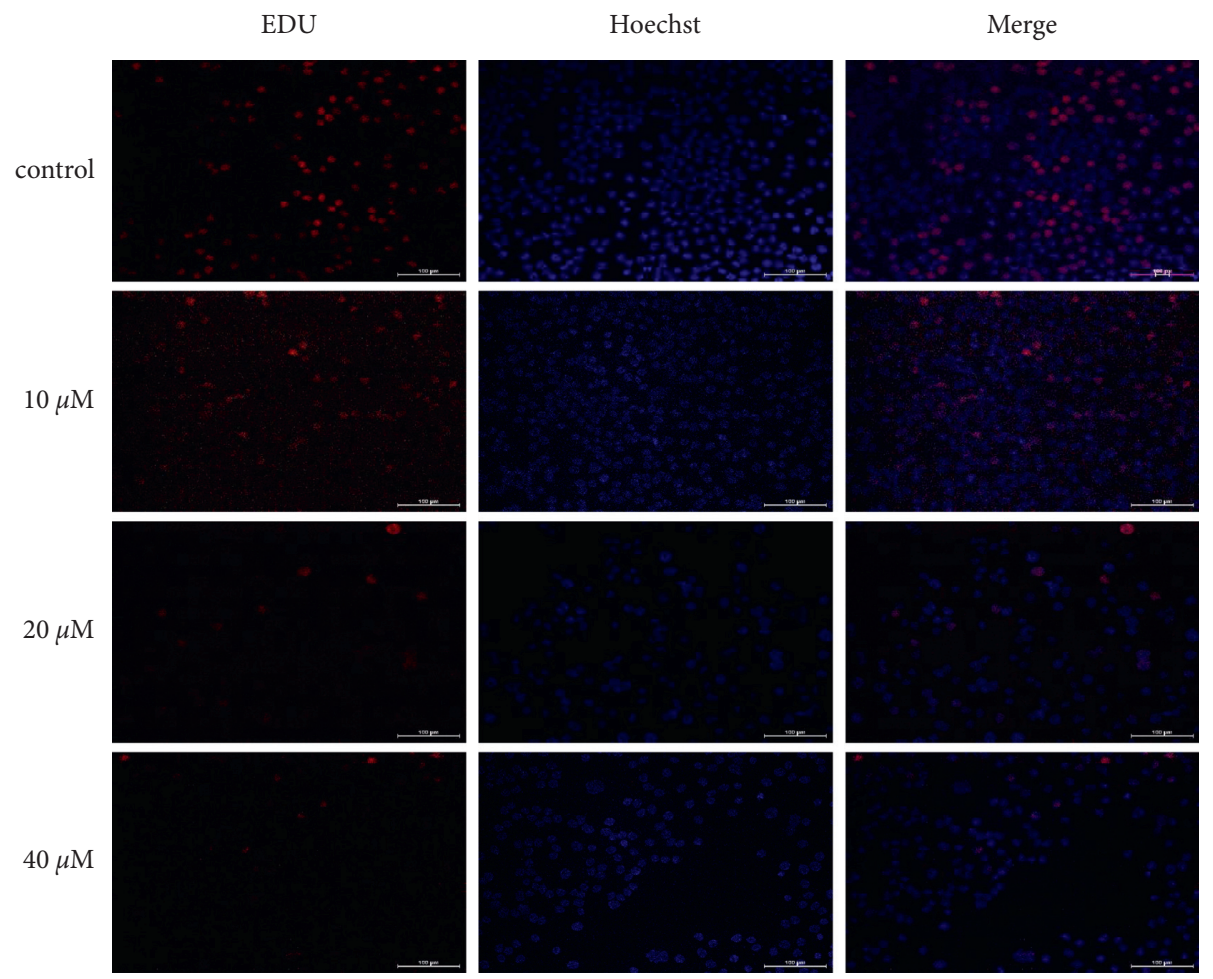

(c)

Figure 2: Continued. 


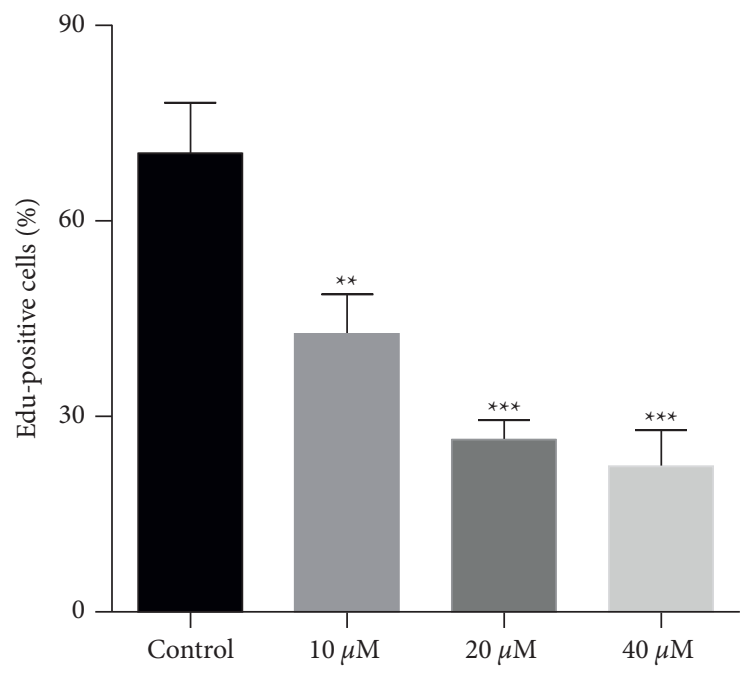

(d)

FIgure 2: CA inhibits proliferation of KYSE-150 cells. (a) Cells were treated with CA and grown for 15 days. CA dose-dependently decreased colony formation. (b) Quantification of colony formation in (a). (c) Representative images showing EdU-positive cells after CA treatment for $48 \mathrm{~h}$. (d) Quantification of (c). The statistical significance was calculated using the unpaired student's two-tailed $t$-test with the $p$ values $\left({ }^{* *} p<0.01\right.$ and ${ }^{* * *} p<0.001$ vs control group).
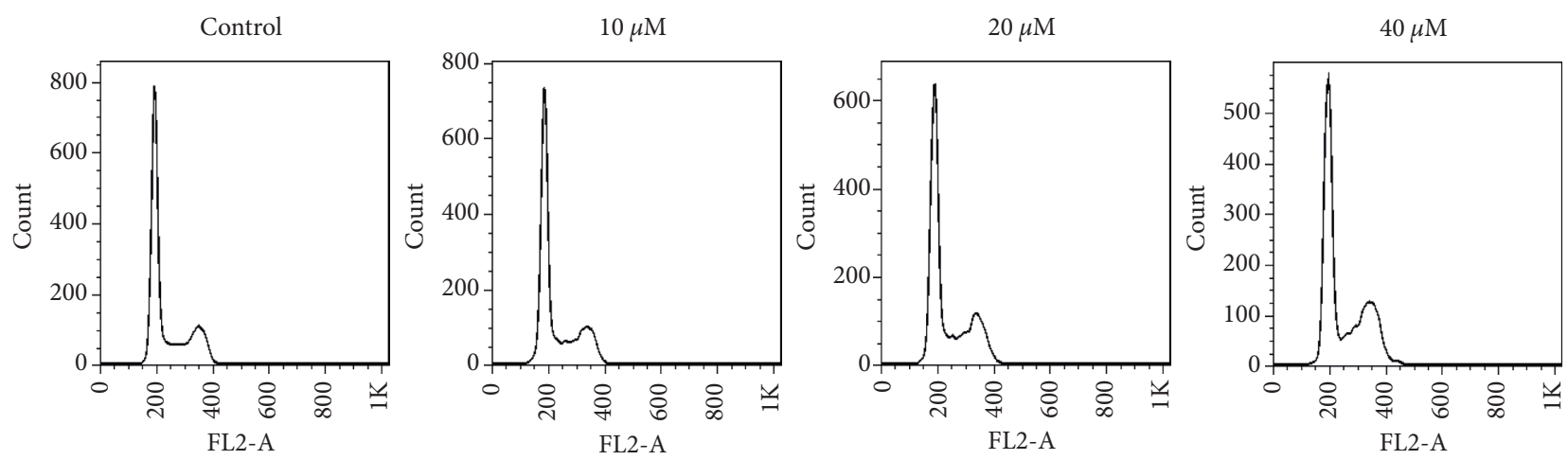

(a)

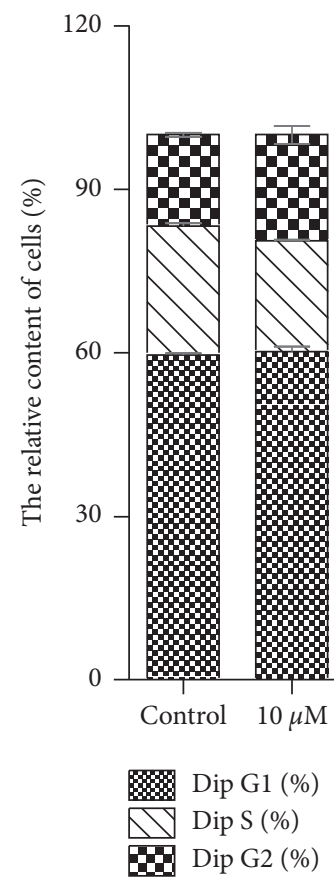

(b)

FIgURE 3: Continued. 


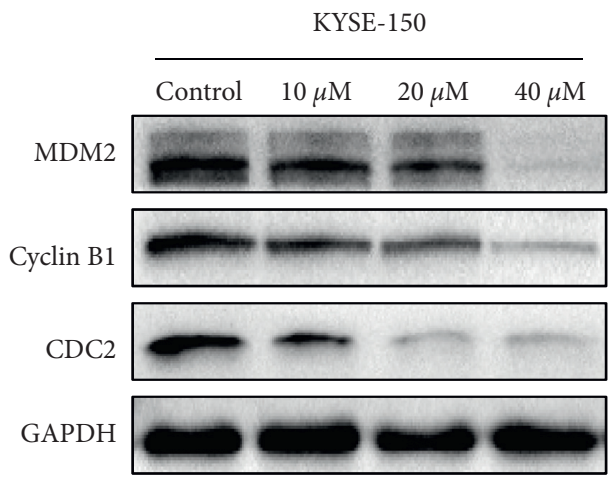

(c)

FIgURE 3: CA arrests KYSE-150 cells at the G2/M phase. KYSE- 150 cells were treated with different concentrations of CA for $48 \mathrm{~h}$. (a) The cell cycle was measured using flow cytometry. (b) Quantification of flow cytometry data in (a). (c) The expressions of MDM2, cyclin B1, and CDC2 were measured using Western blot. Values were mean \pm SD of at least three independent experiments $\left({ }^{*} p<0.05\right.$ and ${ }^{* *} p<0.01$ vs control group).

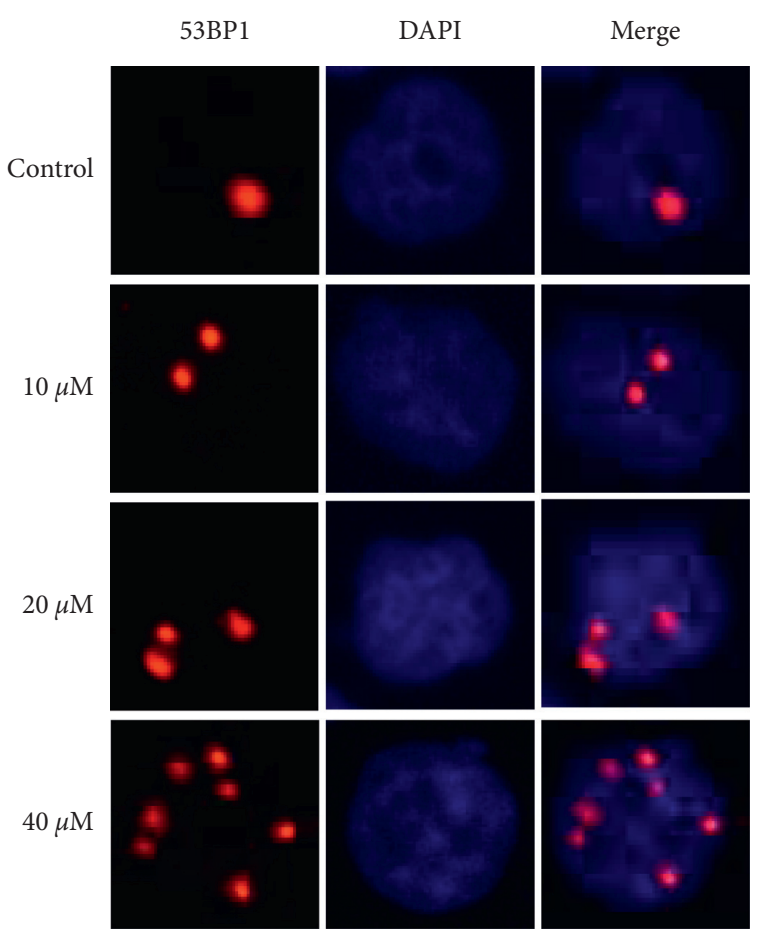

(a)

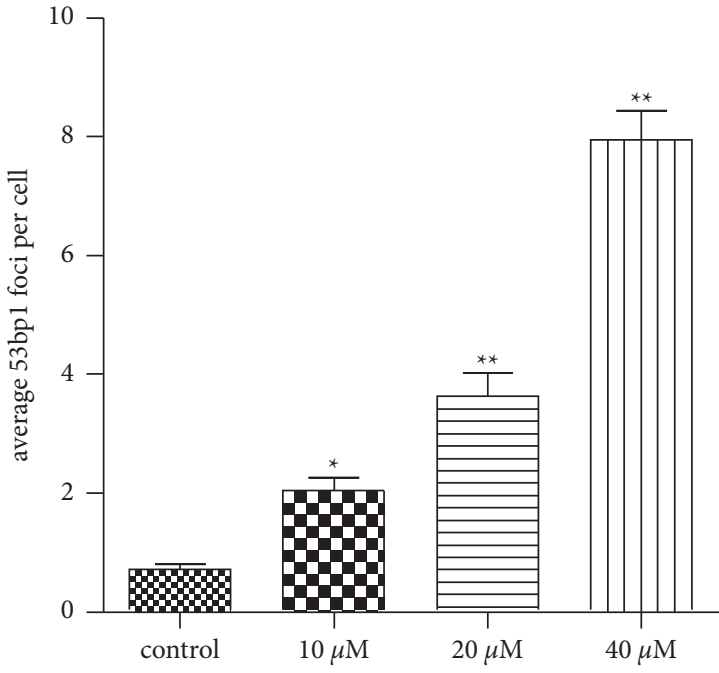

(b)

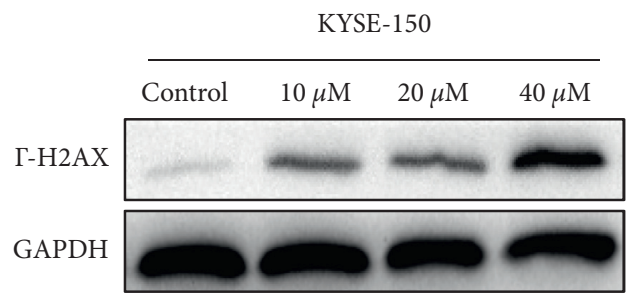

(c)

FIgURE 4: CA provokes intense DNA damage response in KYSE-150 cells. (a) Representative images showing CA treatment provokes intense DNA damage response by immunofluorescence. (b) Quantification of (a) showing that CA dose-dependently increased the DNA damage (more than 200 cells were examined in each group). (c) Western blot showing that CA dose-dependently increased the expression of $\gamma$-H2AX. Values were presented as the mean \pm SD of at least three independent experiments $\left({ }^{*} p<0.05\right.$ and ${ }^{* *} p<0.01$ vs control group). 


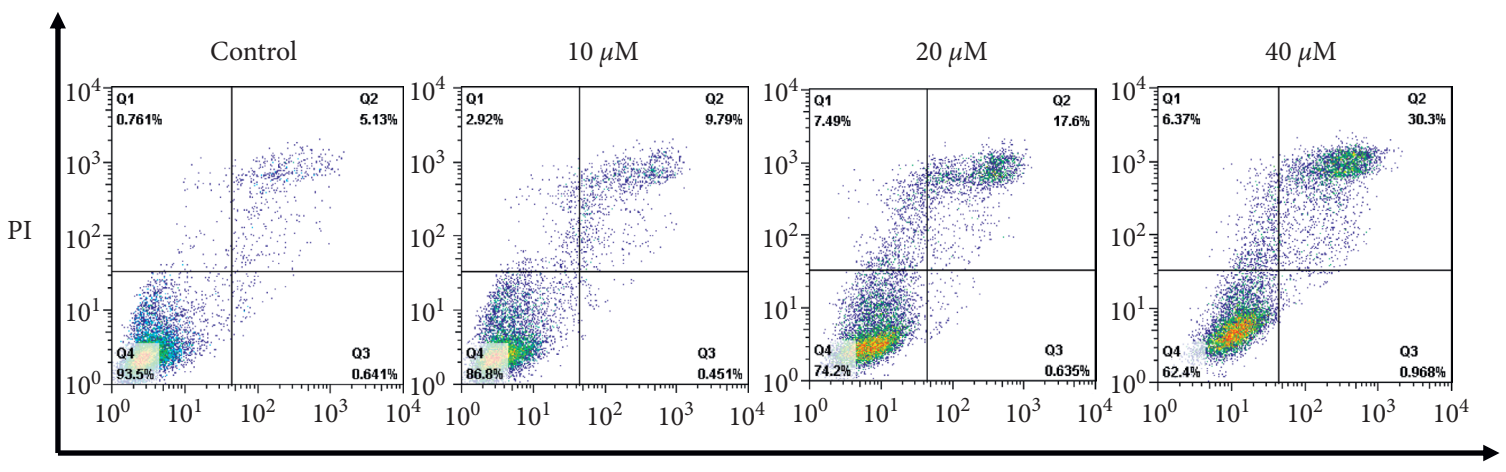

Annexin V

(a)

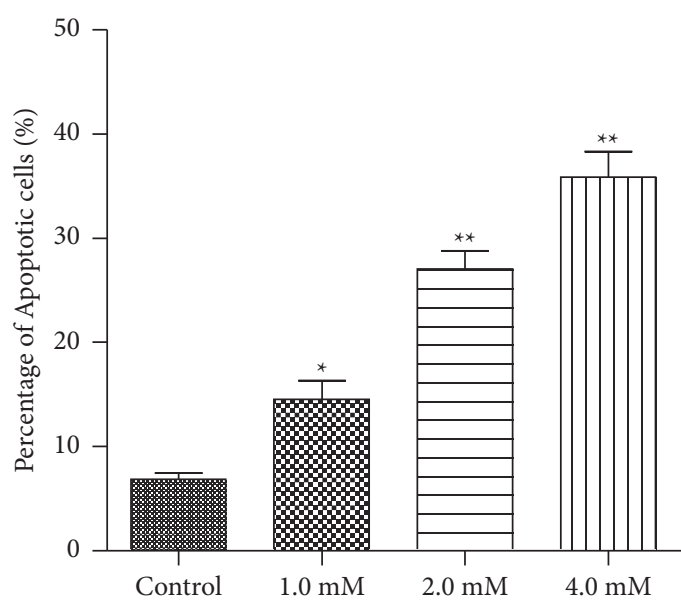

(b)

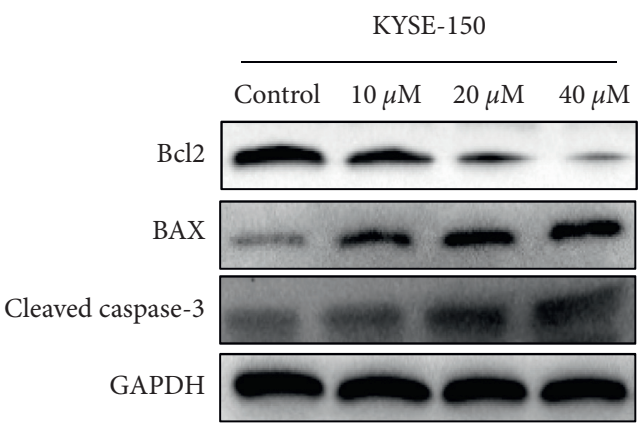

(c)

FIgURE 5: CA promotes apoptosis of KYSE-150 cells. (a) The apoptosis of KYSE-150 was analyzed by flow cytometry. (b) Quantification of flow cytometry data in (a). (c) The expression of Bcl2 was inhibited, while that of Bax and cleaved caspase-3 was activated in a dosedependent manner by Western blot. GAPDH was used as loading control. Values are average \pm SD of three independent experiments $\left({ }^{*} p<0.05\right.$ and ${ }^{* *} p<0.01$ vs control group).

Control

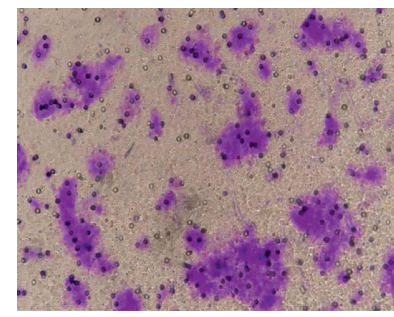

$10 \mu \mathrm{M}$

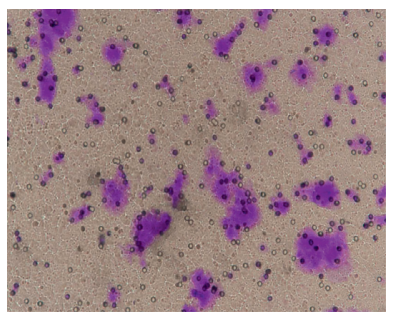

$20 \mu \mathrm{M}$

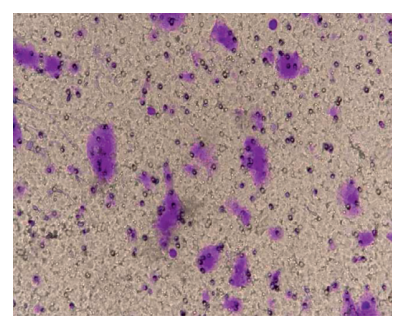

(a)
$40 \mu \mathrm{M}$

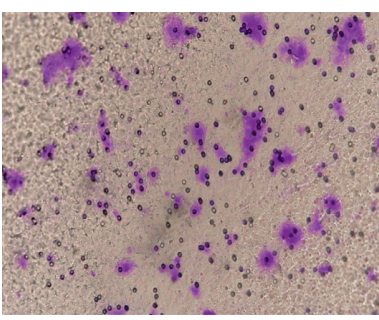

Figure 6: Continued. 


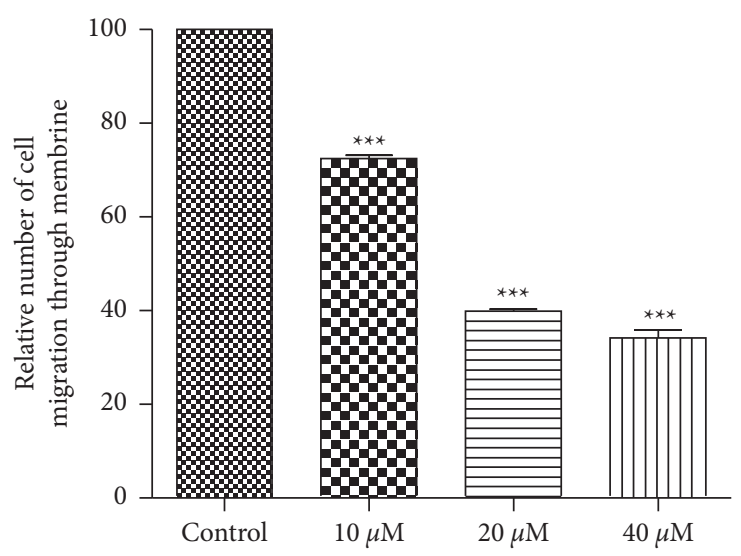

(b)

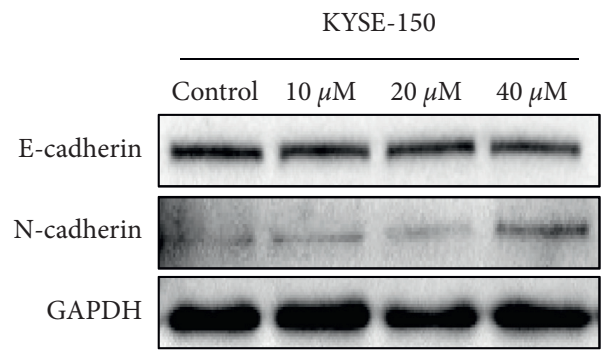

(c)

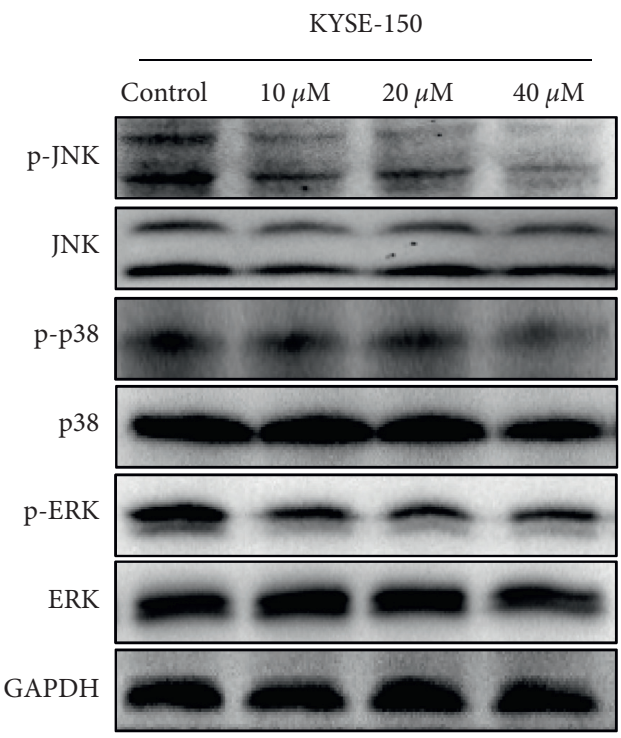

(d)

Figure 6: CA inhibits the migration and invasion of KYSE-150 cells via suppressing the MAPK signaling pathway. (a) CA dose-dependently inhibits cell migration as evaluated by Transwell assay. (b) Quantification of cell migration in the Transwell assay in (a). (c) CA dosedependently decreases the expression E-cadherin and simultaneously increases the expression of $\mathrm{N}$-cadherin, respectively, by Western blot. (d) CA dose-dependently decreases the levels of p-JNK, p-ERK, and p-38 but does not affect those of total JNK, p-38, and ERK by Western blot.

\section{Conclusion}

CA dose-dependently inhibited the malignant phenotypes of an ESCC cell line, KYSE-150, which is most likely mediated by multiple molecular mechanisms, including cell cycle arrest, promotion of apoptosis, inhibition of cell migration, and a coordinated inhibition of ERK, p-38, and JNK signaling pathways. Our observations suggest that CA could be an efficacious drug for ESCC treatment; however, additional testing is warranted in large animal models and clinical trials. 


\section{Abbreviations}

CA: Carnosic acid

ESCC: Esophageal squamous cell carcinoma

EAC: Esophageal adenocarcinoma

EMT: Epithelial-to-mesenchymal transition

DD: Double distilled

IF: Immunofluorescence

CIS: Cisplatin

5-FU: 5-Fluorouracil.

\section{Data Availability}

The data that support the findings of this study will be made available upon request to the corresponding author.

\section{Conflicts of Interest}

The authors declare no conflicts of interest.

\section{Authors' Contributions}

Sicong Jiang and Yinda Qiu contributed equally to this work.

\section{Acknowledgments}

This study was supported by the Jiangxi Key Technology R\&D Program Foundation (2009BSB11003).

\section{References}

[1] B. Jiang, Y. Zhao, M. Shi et al., "DNAJB6 promotes ferroptosis in esophageal squamous cell carcinoma," Digestive Diseases and Sciences, vol. 65, no. 7, pp. 1999-2008, 2020.

[2] Y. Zheng, Z. Chen, Y. Han et al., "Immune suppressive landscape in the human esophageal squamous cell carcinoma microenvironment," Nature Communications, vol. 11, no. 1, p. 6268,2020 .

[3] H. Zhu, H. Jin, J. Pi et al., "Apigenin induced apoptosis in esophageal carcinoma cells by destruction membrane structures," Scanning, vol. 38, no. 4, pp. 322-328, 2016.

[4] Y.-M. Gu, Y. Zhuo, L.-Q. Chen, and Y. Yuan, "The clinical application of neoantigens in esophageal cancer," Frontiers in oncology, vol. 11, p. 703517, 2021.

[5] C. Sang, C. Chao, M. Wang, Y. Zhang, G. Luo, and X. Zhang, "Identification and validation of hub microRNAs dysregulated in esophageal squamous cell carcinoma," Aging, vol. 12, no. 10, pp. 9807-9824, 2020.

[6] M. Sohda and H. Kuwano, "Current status and future prospects for esophageal cancer treatment," Annals of Thoracic and Cardiovascular Surgery, vol. 23, no. 1, pp. 1-11, 2017.

[7] Y. Baba, D. Nomoto, K. Okadome et al., "Tumor immune microenvironment and immune checkpoint inhibitors in esophageal squamous cell carcinoma," Cancer Science, vol. 111, no. 9, pp. 3132-3141, 2020.

[8] A. Fatehi Hassanabad, R. Chehade, D. Breadner, and J. Raphael, "Esophageal carcinoma: towards targeted therapies," Cellular Oncology, vol. 43, no. 2, pp. 195-209, 2020.

[9] W. Liu, T.-C. Wu, D.-M. Hong et al., "Carnosic acid enhances the anti-lung cancer effect of cisplatin by inhibiting myeloidderived suppressor cells," Chinese Journal of Natural Medicines, vol. 16, no. 12, pp. 907-915, 2018.
[10] Y. Liu, Y. Zhang, M. Hu, Y. Li, and X. Cao, "Carnosic acid alleviates brain injury through NF- $\kappa \mathrm{B}$-regulated inflammation and Caspase-3-associated apoptosis in high fat-induced mouse models," Molecular Medicine Reports, vol. 20, no. 1, pp. 495-504, 2019.

[11] J. Moore, M. Yousef, and E. Tsiani, "Anticancer effects of rosemary (rosmarinus officinalis L.) extract and rosemary extract polyphenols," Nutrients, vol. 8, p. 11, 2016.

[12] L. Magnelli, N. Schiavone, F. Staderini, A. Biagioni, and L. Papucci, "MAP kinases pathways in gastric cancer," International Journal of Molecular Sciences, vol. 21, no. 8, 2020.

[13] P. Boueroy, S. Saensa-Ard, P. Siripong, S. Kanthawong, and C. Hahnvajanawong, "Rhinacanthin-C extracted from rhinacanthus nasutus (L.) inhibits cholangiocarcinoma cell migration and invasion by decreasing MMP-2, uPA, FAK and MAPK pathways," Asian Pacific Journal of Cancer Prevention, vol. 19, no. 12, pp. 3605-3613, 2018.

[14] M. Ma, L. Tao, and A. Liu, "Macrophage migration inhibitory factor-794 CATT microsatellite polymorphism and risk of tuberculosis: a meta-analysis," Bioscience Reports, vol. 38, no. 4, 2018.

[15] Y. Wu, S. Lin, Z. Din, J. Su, and C. Liu, "Sinulariolide inhibits gastric cancer cell migration and invasion through downregulation of the EMT process and suppression of FAK/PI3K/ AKT/mTOR and MAPKs signaling pathways," Marine Drugs, vol. 17, no. 12, 2019.

[16] L. Liu, Q.-M. Yin, X. Yan et al., "Bioactivity-guided isolation of cytotoxic phenanthrenes from spiranthes sinensis," Journal of Agricultural and Food Chemistry, vol. 67, no. 26, pp. 7274-7280, 2019.

[17] Z. He, J. Chen, X. Chen, H. Wang, L. Tang, and C. Han, "microRNA-377 acts as a suppressor in esophageal squamous cell carcinoma through CBX3-dependent P53/P21 pathway," Journal of Cellular Physiology, vol. 236, no. 1, pp. 107-120, 2021.

[18] Z. Zheng, P. Yang, and W. Luo, "STAT3 $\beta$ enhances sensitivity to concurrent chemoradiotherapy by inducing cellular necroptosis in esophageal squamous cell carcinoma," Cancers, vol. 13, no. 4, 2021.

[19] H. Huang, Q. Xue, and X. Du, "p21-activated kinase 4 promotes the progression of esophageal squamous cell carcinoma by targeting LASP1," Molecular Carcinogenesis, vol. 60, 2020.

[20] E. Fouquerel, J. Lormand, A. Bose et al., "Oxidative guanine base damage regulates human telomerase activity," Nature Structural \& Molecular Biology, vol. 23, no. 12, pp. 1092-1100, 2016.

[21] D. Lee and H. Jang, "Carnosic acid attenuates an early increase in ROS levels during adipocyte differentiation by suppressing translation of Nox4 and inducing translation of antioxidant enzymes," International Journal of Molecular Sciences, vol. 22, no. 11, 2021.

[22] Y.-M. Yu, H.-C. Lin, and W.-C. Chang, "Carnosic acid prevents the migration of human aortic smooth muscle cells by inhibiting the activation and expression of matrix metalloproteinase-9," British Journal of Nutrition, vol. 100, no. 4, pp. 731-738, 2008.

[23] J. J. Johnson, "Carnosol: a promising anti-cancer and antiinflammatory agent," Cancer Letters, vol. 305, no. 1, pp. 1-7, 2011.

[24] J. J. Johnson, D. N. Syed, Y. Suh et al., "Disruption of androgen and estrogen receptor activity in prostate cancer by a novel dietary diterpene carnosol: implications for chemoprevention," Cancer Prevention Research, vol. 3, no. 9, pp. 1112-1123, 2010. 
[25] M. V. Barni, M. J. Carlini, E. G. Cafferata, L. Puricelli, and S. Moreno, "Carnosic acid inhibits the proliferation and migration capacity of human colorectal cancer cells," Oncology Reports, vol. 27, no. 4, pp. 1041-1048, 2012.

[26] K. Lin, C. Lin, and S. Kuo, "Carnosic acid impedes cell growth and enhances anticancer effects of carmustine and lomustine in melanoma," Bioscience Reports, vol. 38, no. 4, 2018.

[27] L. S. Einbond, H.-a. Wu, R. Kashiwazaki et al., "Carnosic acid inhibits the growth of ER-negative human breast cancer cells and synergizes with curcumin," Fitoterapia, vol. 83, no. 7, pp. 1160-1168, 2012.

[28] S. Kar, S. Palit, W. B. Ball, and P. K. Das, "Carnosic acid modulates Akt/IKK/NF- $\kappa$ B signaling by PP2A and induces intrinsic and extrinsic pathway mediated apoptosis in human prostate carcinoma PC-3 cells," Apoptosis, vol. 17, no. 7, pp. 735-747, 2012.

[29] S. Park, H. Song, M.-K. Sung, Y.-H. Kang, K. Lee, and J. Park, "Carnosic acid inhibits the epithelial-mesenchymal transition in B16F10 melanoma cells: a possible mechanism for the inhibition of cell migration," International Journal of Molecular Sciences, vol. 15, no. 7, pp. 12698-12713, 2014.

[30] K.-J. Jung, K.-j. Min, J. H. Bae, and T. K. Kwon, "Carnosic acid sensitized TRAIL-mediated apoptosis through down-regulation of c-FLIP and Bcl-2 expression at the post translational levels and CHOP-dependent up-regulation of DR5, Bim, and PUMA expression in human carcinoma caki cells," Oncotarget, vol. 6, no. 3, pp. 1556-1568, 2015.

[31] X. Zhou, Y. Kuang, S. Liang, and L. Wang, "Metformin inhibits cell proliferation in SKM-1 cells via AMPK-mediated cell cycle arrest," Journal of Pharmacological Sciences, vol. 141, no. 4, pp. 146-152, 2019.

[32] Y. Wang, W. Xu, and Z. Yan, "Metformin induces autophagy and G0/G1 phase cell cycle arrest in myeloma by targeting the AMPK/mTORC1 and mTORC2 pathways," Journal of Experimental \& Clinical Cancer Research: Climate Research, vol. 37 , no. 1, p. 63, 2018.

[33] T.-W. Chung, S.-C. Lin, J.-H. Su, Y.-K. Chen, C.-C. Lin, and H.-L. Chan, "Sinularin induces DNA damage, G2/M phase arrest, and apoptosis in human hepatocellular carcinoma cells," BMC Complementary and Alternative Medicine, vol. 17, no. 1, p. 62, 2017.

[34] H.-J. Eom and J. Choi, "p38 MAPK activation, DNA damage, cell cycle arrest and apoptosis as mechanisms of toxicity of silver nanoparticles in Jurkat T cells," Environmental Science \& Technology, vol. 44, no. 21, pp. 8337-8342, 2010.

[35] C.-Y. Yu, C.-L. Jerry Teng, P.-S. Hung et al., "Ovatodiolide isolated from Anisomeles indica induces cell cycle G2/M arrest and apoptosis via a ROS-dependent ATM/ATR signaling pathways," European Journal of Pharmacology, vol. 819, pp. 16-29, 2018.

[36] J. H. Tsai and J. Yang, "Epithelial-mesenchymal plasticity in carcinoma metastasis," Genes \& Development, vol. 27, no. 20, pp. 2192-2206, 2013. 